This document is confidential and is proprietary to the American Chemical Society and its authors. Do not copy or disclose without written permission. If you have received this item in error, notify the sender and delete all copies.

\title{
Peptides Derived from Soy and Lupin Protein as Dipeptidyl- peptidase IV Inhibitors: In Vitro Screening and In Silico Molecular Modelling Study
}

\begin{tabular}{|r|l|}
\hline Journal: & Journal of Agricultural and Food Chemistry \\
\hline Manuscript ID & Draft \\
\hline Manuscript Type: & Article \\
\hline Date Submitted by the Author: & $\mathrm{n}$ a \\
\hline Complete List of Authors: & $\begin{array}{l}\text { Lammi, Carmen; University of Milan, Pharmaceutical Sciences } \\
\text { Zanoni, Chiara; Universita degli Studi di Milano, Department of } \\
\text { Pharmaceutical Sciences } \\
\text { Arnoldi, Anna; University of Milan, Pharmaceutical Sciences } \\
\text { Vistoli, Giulio; University of Milan, Department of Pharmaceutical Sciences } \\
\text { P. Pratesi }\end{array}$ \\
\hline
\end{tabular}




\section{Peptides Derived from Soy and Lupin Protein as Dipeptidyl-peptidase}

2 IV Inhibitors: In Vitro Screening and In Silico Molecular Modelling

3 Study

4

5

6

Carmen Lammi, Chiara Zanoni, Anna Arnoldi, * Giulio Vistoli

Department of Pharmaceutical Sciences, University of Milan, Milan, Italy

*Corresponding author: Anna Arnoldi, Department of Pharmaceutical Sciences, University of Milan, via Mangiagalli 25, 20133 Milan, Italy. Tel +390250319372, fax +390250319343; e-mail anna.arnoldi@unimi.it

\section{Abstract}

Dipeptidyl peptidase IV (DPP IV) is a new molecular target correlated with the development of type 2 diabetes. Literatures describes the identification of some inhibitory peptides from the hydrolysis of different food proteins. This paper reports a study on six peptides from soybean and lupin proteins, i.e Soy 1 (IAVPTGVA), Soy 2 (YVVNPDNDEN), Soy 3 (YVVNPDNNEN), Lup 1 (LTFPGSAED), Lup 2 (LILPKHSDAD), and Lup 3 (GQEQSHQDEGVIVR), which were screened for their capacity to inhibit this enzyme, using an in vitro bioassay against human recombinant DPP IV. Two peptides Soy 1 and Lup 1 resulted to be efficient inhibitors of DPP IV activity, with $\mathrm{IC}_{50}$ values equal to 106 and $228 \mu \mathrm{M}$, respectively. A molecular docking analysis predicted the key molecular interactions, stabilizing the active peptides within DPP IV enzyme. Soy and lupin proteins are sources of DPP IV inhibitory peptides potentially useful for the prevention of type 2 diabetes.

KEYWORDS: bioactive peptide, dipeptidyl peptidase IV inhibitor, lupin, soy, type 2 diabetes 


\section{INTRODUCTION}

Plant proteins are useful in the prevention of cardiovascular disease and diabetes. ${ }^{l}$ In particular, some studies provide evidence that soy protein and/or peptides exert a hypoglycemic activity either in animals ${ }^{2,3}$ or in type-2 diabetic patients. ${ }^{4,5}$ Moreover, some peptides from soy protein improve glucose uptake in HepG2 cells ${ }^{6}$ and peptide mixtures obtained by pepsin-pancreatin hydrolysis of soy protein improve glucose uptake in muscle L6 cells. ${ }^{7}$ In the meanwhile, other investigations support the hypoglycemic activity of lupin protein. ${ }^{8,9}$ In particular, it has been demonstrated that $\gamma$ conglutin, a sulfur-rich lupin protein, decreases blood glucose concentration in rats ${ }^{10}$ and has a relevant post-prandial hypoglycemic effect in humans. ${ }^{10}$ All these pieces of evidence suggest that the soy and lupin protein consumption may be beneficial for the prevention of type 2 diabetes.

Dipeptidyl peptidase IV (DPP IV) is a new molecular target correlated with the development of diabetes. ${ }^{11}$ DPP IV is a serine exopeptidase that cleaves Xaa-proline or Xaa-alanine dipeptides from the N-terminus of polypeptides. Among all DPP IV substrates, the most widely investigated are glucagon-like peptide 1 (GLP-1) and glucose-dependent insulinotropic polypeptide (GIP), two incretins playing an essential role in maintaining glucose homeostasis. ${ }^{12,13}$ Together, they stimulate the insulin biosynthesis at pancreatic level and are responsible for up to $70 \%$ of insulin secretion following a meal. ${ }^{14,15}$ Since after secretion, GLP-1 and GIP are rapidly degraded by DPP IV, ${ }^{16}$ the inhibition of DPP IV improves the glucose tolerance in diabetic patients by enhancing the insulinotropic effects of GLP- $1^{17}$ and lowers blood glucose via stimulation of insulin and inhibition of glucagon. For this reason DPP IV inhibitors have emerged as a new class of oral antidiabetic agents, ${ }^{18}$ with an excellent therapeutic potential in the management of type 2 diabetes. ${ }^{11,19}$ The synthetic DPP-IV inhibitors sitagliptin and vildagliptin are currently the most widely investigated new drugs for the treatment of type- 2 diabetes. ${ }^{20}$

Interestingly, many food proteins appear to be useful sources of DPP IV inhibitory peptides, which may be released from their sequences by enzymatic hydrolysis. For example, DPP IV inhibitory 
49

50

51

52

53

54

peptides have been isolated and characterized from the proteins of cow milk, ${ }^{21}$ goat milk, ${ }^{22}$ silver carp, ${ }^{23}$ tuna, ${ }^{24}$ salmon, ${ }^{25}$ rice, ${ }^{26}$ black bean, ${ }^{27}$ and amaranth. ${ }^{28}$

Owing to our interest for the role of plant proteins and peptides in the prevention of hypercholesterolemia and hyperglycemia, in the last few years, we have collected some bioactive peptides from soy (Soy 1-3) and lupin proteins (Lup 1-3) and investigated their biological activities (Table 1). Soy 1 (IAVPTGVA) corresponds to position 142-149 of glycinin (UniProtK P04776.2) and modulates cholesterol and glucose biosyntheses in HepG2 cells. ${ }^{6,29,30}$ Soy 2 (YVVNPDNDEN) corresponds to position 232-241 of the $\alpha$ subunit of $\beta$-conglycinin (UNIProtKB P13916), whereas Soy 3 (YVVNPDNNEN) corresponds to positions 310-319 of the $\alpha^{\prime}$ subunit of $\beta$-conglycinin (UNIProtKB P11827). Both are able to inhibit the activity of 3-hydroxymethylglutarylCoA reductase (HMGCoAR) and to modulate cholesterol biosynthesis in HepG2 cells. ${ }^{31}$ Interestingly, an investigation in Caco2 cells has shown that they are potentially absorbed at intestine level. ${ }^{32}$ Lup 1 (LTFPGSAED), Lup 2 (LILPKHSDAD), and Lup 3 (GQEQSHQDEGVIVR) correspond to positions 484-492, 235-244, and 362-375, respectively, of $\beta$-conglutin (UniProtKB Q53HY0.2), a $7 \mathrm{~S}$ storage protein. We have recently demonstrated that they are transferred from the apical to the basolateral chamber of a monolayer of Caco2 cells grown in a bicameral system and that the basolateral solution where they were detected inhibits the activity of HMGCoAR ${ }^{33}$

BIOPEP (www.uwm.edu.pI/biochemia $)^{34}$ is an open access database enabling to hypothesize the potential biological activities of peptides based on the presence of some specific amino acid sequences. A screening of the structures of these soy or lupin peptides with BIOPEP suggested that their structures were compatible with a potential function as DPP IV inhibitors. It was thus decided to evaluate their potential inhibitory activity using a commercial in vitro bioassay against human recombinant DPP IV. Subsequently, the interaction of two active peptides and an inactive one with the enzyme was investigated, by employing an in silico molecular model and scoring approach in order to perform a docking simulation study. The present work describes the results of these investigations. 
76

77

\section{MATERIAL \& METHODS}

Materials. Tris- $\mathrm{HCl}$, ethylenediamine tetra-acetic acid (EDTA), and $\mathrm{NaCl}$ were from SigmaAldrich (St. Louis, MO, USA). The DPP IV enzyme and the substrate solution [5 mM H-Gly-Pro conjugated to aminomethylcoumarin (H-Gly-Pro-AMC)] were provided by Cayman Chemicals (Michigan, USA). The peptides Soy 1-3 and Lup 1-3 (Table 1) were synthesized by the company PRIMM (Milan, Italy) with $>95 \%$ purity assessed by HPLC.

DPP IV activity assay. The DPP IV enzyme and the substrate solution (5 mM H-Gly-Pro-AMC) were provided by Cayman Chemicals (Michigan, USA). The experiments were carried out in triplicate in a half volume 96 well solid plate (white). Each reaction $(50 \mu \mathrm{L})$ was prepared adding the reagents in the following order in a microcentrifuge tube: $1 \mathrm{X}$ assay buffer $[20 \mathrm{mM}$ Tris-HCl, pH 8.0, containing $100 \mathrm{mM} \mathrm{NaCl}$, and $1 \mathrm{mM}$ EDTA] $(30 \mu \mathrm{L}), 100 \mu \mathrm{M}$ of each soy and lupin peptide [Soy 1-3 or Lup 1-3] or vehicle $(10 \mu \mathrm{L})$ and finally the DPP IV enzyme $(10 \mu \mathrm{L})$. Subsequently, the samples were mixed and $50 \mu \mathrm{L}$ of each reaction were transferred in each well of the plate. Each reaction was started by adding $50 \mu \mathrm{L}$ of substrate solution to each well and incubated at $37{ }^{\circ} \mathrm{C}$ for 30 minutes. Fluorescence signals were measured using the Synergy H1 fluorescent plate reader from Biotek (excitation and emission wavelengths 360 and $465 \mathrm{~nm}$, respectively). In order to build the dose-inhibition curves of the active peptides Soy $\mathbf{1}$ and Lup 1, concentrations in the range $10-1000 \mu \mathrm{M}$ were tested using the same procedure described above.

Statistically Analysis. Statistical analyses were carried out by One-way ANOVA using Graphpad Prism 6 (Graphpad, La Jolla, CA, USA) followed by Dunnett's test. Values were expressed as means $\pm \mathrm{SEM} ; P$-values $<0.05$ were considered to be significant. 
Computational methods. By applying a computational strategy already adopted in a previous study, ${ }^{35}$ two active and one inactive peptides (i.e., Soy 1, Lup 1, and Lup 2) were built in canonical $\alpha$-helix by using the Peptide Builder function of the VEGA suite of programs ${ }^{36}$ and then their conformational profiles were explored by a MonteCarlo procedure, which produced 10,000 conformers by randomly rotating the backbone torsions only. The obtained geometries were then clustered according to their similarity to discard redundant ones; here, two conformations were considered as non-redundant when they differed by more than 60 degrees in at least one backbone torsion angle. For each cluster, the lowest energy structure was collected and memorized.

Among the resolved human DPP IV structures, the study involved the complex between the enzyme and the long-acting inhibitor Omarigliptin (PDB Id: 4PNZ) chosen due to its very high resolution ${ }^{37}$. After deleting water molecules, ions and crystallization additives, the selected dimer bound to Omarigliptin was completed by adding the hydrogen atoms and then optimized by keeping fixed the backbone atoms to preserve the resolved folding. The inhibitor was finally deleted and the obtained protein structure underwent the following docking simulations.

Docking simulations were carried out by using PLANTS and involved the 20 lowest energy conformations as derived by the previous MonteCarlo analysis in order to minimize the biasing effects of the starting conformation on the obtained results ${ }^{38}$. In detail, the search was focused on a $12.0 \AA$ radius sphere around the bound Omarigliptin thus including the entire binding cavity. PLANTS was used with default settings and without geometric constraints, speed 1 was used and 5 poses were generated for each conformer and scored by using the PLP function. The obtained poses were evaluated by considering both the docking scores and the conformational energies of the docked conformers. The best generated poses was then minimized keeping fixed all atoms inside a $12.0 \AA$ radius sphere around the bound peptide. 


\section{RESULTS}

Soy and Lupin peptides are able to inhibit DPP IV activity. Figure 1 shows the results of the experiments aimed to evaluate the inhibitory activity of soybean and lupin peptides against recombinant DPP IV using H-Gly-Pro-AMC as substrate. The enzymatic reaction was monitored measuring the fluorescence signals, emitted at $465 \mathrm{~nm}$, due to the free AMC group release after the cleavage of the peptide H-Gly-Pro by DPP IV. Each peptide was screened at the final concentration of $100 \mu \mathrm{M}$, in parallel with the positive control, sitagliptin $(0.1 \mu \mathrm{M})$. Two peptides, one from soy protein and another from lupin protein, were able to inhibit the DPP IV activity: Soy 1 reduced the DPP IV activity by 46\% and Lup 1 by 35\%. On the contrary, Soy 2, Soy 3, Lup 2, and Lup 3 weare inactive, whereas the positive control sitagliptin inhibited the DPP IV activity by $88 \%$ at 0.1 $\mu \mathrm{M}$ (Figure 1). Subsequently, specific dose-response curves were built for Soy 1 and Lup 1 (Figure 2). Soy 1 displayed the highest inhibitory activity with an estimated $\mathrm{IC}_{50}$ value of $106 \mu \mathrm{M}$, whereas Lup 1 was less efficient, since its $\mathrm{IC}_{50}$ was $228 \mu \mathrm{M}$.

Molecular modeling investigation. Figure 3 shows the putative complex between Soy 1 and DPP IV revealing the key ionic interactions, which involve both peptide charged termini and seem to play a largely predominant role. In detail, the amino terminus is engaged in a double salt bridge involving Glu205 and Glu206, while the carboxyl terminus stabilizes an ion-pair with Arg358. Apart from Thr5, which reinforces the contacts elicited by the ammonium head by approaching Glu205, the remaining part of the peptide appears to be marginally involved in the complex stabilization. In fact, the central residues might even play a negative role, since peptide apolar residues are seen to contact protein polar residues as in the case of Pro4, which unfittingly approaches Glu206 and Ser209. Moreover, Soy 1 does not contains any aromatic side-chains and thus cannot elicit $\pi-\pi$ stacking interactions with the numerous aromatic residues lining the enzyme cavity. 
This pattern of key interactions can easily rationalize the different inhibitory activity observed for the other simulated peptides. In detail, the marked difference in the inhibition activity between Lup 2 and Lup 1 seems to be mostly ascribable to the interfering effect of the central Lys5 residue in Lup 2, which stabilizes the ionic contacts normally involving the amino terminus, which is therefore constrained to detrimentally approach Arg125. In detail, the carboxyl terminus of both Lup 2 and Lup 1 peptides interacts with Arg358 and Arg356 and is engaged in an extended ionic network also involving the side-chain of the C-terminal residue (Asp10 in Lup 2 and Asp9 in Lup 1) and Arg429. As mentioned above, the greatest differences concern the contacts stabilized by the amino terminus, since it elicits the already described ion-pairs with Glu205 and Glu206 in the active peptide Lup 1, whereas in the inactive Lup 2 peptide it is replaced by Lys5 and is confined to a lateral pose where it approaches Arg125, while contacting Glu205. In the putative complexes of both Lup 2 and Lup 1, the central residues seems to play non-negligible roles. In detail, the negatively charged residue in the C-terminal segment of both peptides (Asp8 of Lup 2 and Glu8 of Lup 1) are involved in the above described ionic network stabilized around the carboxyl terminus. More importantly, Lup 1 includes an aromatic residue (Phe3), which is engaged in a rich set of $\pi-\pi$ stacking involving Tyr547, Trp629, and His740.

Taken together, the docking results allow some general considerations. Ionic interactions stabilized by charged termini play a clearly crucial role even though their contribution is easily saturating and the stabilizing effect of the additional ionized side chains appears to be almost negligible, if not even negative (as seen for Lys5 in Lup 2). This effect can be explained by considering the closeness between the cluster of protein negatively charged residues (i.e. Glu205 and Glu206) and that of positively charged residues (i.e. Arg358 and Arg356) and more generally the richness of ionized residues lining the enzyme cavity. In this way, the additional ionized side chains tend to interfere with the crucial contacts elicited by charged termini rather than playing a concrete stabilizing role. 
Clearly, such an interfering effect is an indirect consequence of the molecular size of the simulated peptides, which are excessively bulky when considering that the enzyme cavity is arranged to accommodate dipeptide substrates. The unsuitable length can thus explain the inactivity of the other (non-simulated) longer peptides (i.e. Soy 2, Soy 3, and Lup 3) and can surely contribute to the inactivity of Lup 2. Finally, the non-ionized central residues of the simulated peptides appears to play very negligible roles and probably only Phe3 of Lup 1, which is engaged in a rich set of $\pi-\pi$ stacking interactions, should have a concrete stabilizing function thus differentiating Lup 1 from the other considered ligands.

\section{DISCUSSION}

Although the health benefits of soy and lupin protein consumption are well known, particularly in the area of cholesterol reduction, hypertension, and hyperglycaemia prevention, this is the first study providing evidence that some peptides from soy and lupin protein, i.e. Soy $\mathbf{1}$ and Lup 1, are able to inhibit the DPP IV activity. Our experimentation suggests a new mechanism of action through which soy and lupin protein may mediate some health benefits in the area of hyperglycemia prevention.

Some years ago, a patent ${ }^{39}$ has reported the structures of 21 peptides capable of inhibiting DPP IV activity. They have a hydrophobic character, a length varying from 2 to 8 amino acid residues, and contain a Pro residue within their sequences, which is located at the first, second, third, or fourth Nterminal position. Besides, the Pro residue is flanked by Leu, Val, Phe, Ala, and Gly. Indeed, our data are consistent with this patent. In fact, as the fourth N-terminal residue, the active peptides Soy $\mathbf{1}$ and Lup 1 comprise a Pro, which is flanked by a Val residue in Soy $\mathbf{1}$ and by a Phe residue in Lup 1. Moreover, the peptides are mostly composed of hydrophobic amino acid residues, such as Ala, Gly, Ile, Leu, and Pro. The inactive peptides, i.e. Soy 2, Soy 3, Lup 2 and Lup 3, are probably too long, since they contain 10-14 amino acid residues. In addition, some of them do not respect the 
structural indicated features: Soy 2 and Soy 3 comprise a Pro residue unfavorably located as fifth Nterminal residue and not flanked by any hydrophobic amino acid residue, whereas Lup 3 does not contain any Pro residue.

Finally, it is useful to compare the DPP IV inhibitory activities of our peptides with those of peptides from other foods, such silver carp protein, ${ }^{23}$ Atlantic salmon skin gelatin, ${ }^{25}$ and goat milk protein. $^{22}$ Four peptides (AGPPGPSG, APGPAGP, LPIIDI, and ALAPSTM) have been identified from the hydrolysis of silver carp protein; ${ }^{23}$ out of them LPIIDI and APGPAGP showed the highest DPP IV inhibitory activity, with $\mathrm{IC}_{50}$ values equal to 105.4 and $229.1 \mu \mathrm{M}$, respectively, ${ }^{23}$ which are similar to those of Soy 1 and Lup 1. On the contrary, the peptides GPAG and GPGA from Atlantic salmon skin gelatin ${ }^{25}$ and AWPQYL and INNQFLPYPY from goat milk ${ }^{22}$ appeared to be more active, showing the following $\mathrm{IC}_{50}$ inhibitory values: GPAG $\mathrm{IC}_{50}=49.6 \mu \mathrm{M}, \quad \mathrm{GPGA} \mathrm{IC}_{50}=41.9$ $\mu \mathrm{M}$, AWPQYL $\mathrm{IC}_{50}=40.1 \mu \mathrm{M}$ and INNQFLPYPY $\mathrm{IC}_{50}=40.1 \mu \mathrm{M}$.

When discussing the relevance of the activity of any food component, a general issue is the bioavailability. In this case, the situation appears to be particularly favorable for Lup 1, since a very recent paper has already demonstrated that this peptide is able to across a monolayer of differentiated human enterocytes (CaCo-2 cells), ${ }^{33}$ an in vitro model of gastrointestinal absorption. Work is in progress in our lab to assess the bioavailability of Soy $\mathbf{1}$.

\section{Author Contributions}

Experiment ideation and design: CL and GV. Experiments \& data analysis: biotechnology CL \& CZ; molecular modeling GV. Figure preparation: GV and CZ. Grant retrieval: AA. Manuscript writing: CL, GV \& AA.

\section{Funding}

Researches funded in part by the European Union Seventh Framework Program (FP7/2007-2013), under grant agreement n. 285819. We are indebted to Alpro Foundation for funding of a postdoc 
225 fellowship to CZ, and to Carlo Sirtori Foundation (Milan, Italy) for having provided part of 226 equipment used in this experimentation.

227

228

Notes

229 The authors declare no competing financial interest.

230

231

232

233

234

235

236

237

238

239

240

241

242

243

244

245

246

\section{ABBREVIATIONS USED}

DPP IV, dipeptidyl peptidase IV, GLP-1, glucagon-like peptide 1; GIP, glucose-dependent insulinotropic polypetide; AMC,.aminomethylcoumarin; EDTA, Ethylenediamine tetra-acetic acid.

\section{REFERENCES}

1. Arnoldi, A.; Zanoni, C.; Lammi, C.; Boschin, G., The role of grain legumes in the prevention of hypercholesterolemia and hypertension. Critic Rev Plant Sci 2015, 34, 144-168.

2. Ishihara, K.; Oyaizu, S.; Fukuchi, Y.; Mizunoya, W.; Segawa, K.; Takahashi, M.; Mita, Y.; Fukuya, Y.; Fushiki, T.; Yasumoto, K., A soybean peptide isolate diet promotes postprandial carbohydrate oxidation and energy expenditure in type II diabetic mice. $J$ Nutr 2003, 133, 752-7.

3. Oliva, M. E.; Selenscig, D.; D'Alessandro, M. E.; Chicco, A.; Lombardo, Y. B., Soya protein ameliorates the metabolic abnormalities of dysfunctional adipose tissue of dyslipidaemic rats fed a sucrose-rich diet. Br J Nutr 2011, 105, 1188-98.

4. Anderson, J. W.; Blake, J. E.; Turner, J.; Smith, B. M., Effects of soy protein on renal function and proteinuria in patients with type 2 diabetes. Am J Clin Nutr 1998, 68, 1347S-1353S. 
247 5. Dove, E. R.; Mori, T. A.; Chew, G. T.; Barden, A. E.; Woodman, R. J.; Puddey, I. B.;

248 Sipsas, S.; Hodgson, J. M., Lupin and soya reduce glycaemia acutely in type 2 diabetes. Br J Nutr 249 2011, 106, 1045-51.

250 6. Lammi, C.; Zanoni, C.; Arnoldi, A., Three peptides from soy glycinin modulate glucose 251 metabolism in human hepatic HepG2 cells. Int J Mol Sci 2015, 16, 27362-70.

252 7. Roblet, C.; Doyen, A.; Amiot, J.; Pilon, G.; Marette, A.; Bazinet, L., Enhancement of 253 glucose uptake in muscular cell by soybean charged peptides isolated by electrodialysis with 254 ultrafiltration membranes (EDUF): activation of the AMPK pathway. Food Chem 2014, 147, $124-$ 25530.

8. Duranti, M.; Consonni, A.; Magni, C.; Sessa, F.; Scarafoni, A., The major proteins of lupin 257 seed: characterisation and molecular properties for use as functional and nutraceutical ingredients. 258 Trends Food Sci Technol 2008, 19, 624-633.

9. Arnoldi, A.; Boschin, G.; Zanoni, C.; Lammi, C., The health benefits of sweet lupin seed 260 flours and isolated proteins. J Funct Foods 2015, 18, 550-563.

10. Bertoglio, J. C.; Calvo, M. A.; Hancke, J. L.; Burgos, R. A.; Riva, A.; Morazzoni, P.; 262 Ponzone, C.; Magni, C.; Duranti, M., Hypoglycemic effect of lupin seed gamma-conglutin in 263 experimental animals and healthy human subjects. Fitoterapia 2011, 82, 933-938.

264 11. Bailey, C. J.; Tahrani, A. A.; Barnett, A. H., Future glucose-lowering drugs for type 2 265 diabetes. Lancet Diabetes Endocrinol 2016, 4, 350-359. physiological incretin in man. Lancet 1987, 2, 1300-4. 
268

13. Dupre, J.; Ross, S. A.; Watson, D.; Brown, J. C., Stimulation of insulin secretion by gastric inhibitory polypeptide in man. J Clin Endocrinol Metab 1973, 37, 826-8.

14. Nauck, M. A.; Baller, B.; Meier, J. J., Gastric inhibitory polypeptide and glucagon-like peptide-1 in the pathogenesis of type 2 diabetes. Diabetes 2004, 53 Suppl 3, S190-6.

15. Lynn, F. C.; Pamir, N.; Ng, E. H.; McIntosh, C. H.; Kieffer, T. J.; Pederson, R. A., Defective glucose-dependent insulinotropic polypeptide receptor expression in diabetic fatty Zucker rats. Diabetes 2001, 50, 1004-11.

16. Doupis, J.; Veves, A., DPP4 inhibitors: a new approach in diabetes treatment. Adv Ther 2008, $25,627-43$

17. Ahrén, B.; Gomis, R.; Standl, E.; Mills, D.; Schweizer, A., Twelve- and 52-week efficacy of the dipeptidyl peptidase IV inhibitor LAF237 in metformin-treated patients with type 2 diabetes. Diabetes Care 2004, 27, 2874-80.

18. Carr, R. D., Drug development from the bench to the pharmacy: with special reference to dipeptidyl peptidase-4 inhibitor development. Diabet Med 2016, doi: 10.1111/dme.13066.

19. Ahrén, B.; Foley, J. E., Improved glucose regulation in type 2 diabetic patients with DPP-4 inhibitors: focus on alpha and beta cell function and lipid metabolism. Diabetologia 2016, 5, 90717.

20. Fisman, E. Z.; Tenenbaum, A., Antidiabetic treatment with gliptins: focus on cardiovascular effects and outcomes. Cardiovasc Diabetol 2015, 14, 129.

21. Lacroix, I. M. E.; Li-Chan, E. C. Y., Dipeptidyl peptidase-IV inhibitory activity of dairy protein hydrolysates. International Dairy Journal 2012, 25, 97-102. 
22. Zhang, Y.; Chen, R.; Ma, H.; Chen, S., Isolation and identification of dipeptidyl peptidase iv-inhibitory peptides from trypsin/chymotrypsin-treated goat milk casein hydrolysates by 2D-TLC and LC-MS/MS. J Agric Food Chem 2015, 63, 8819-28.

23. Zhang, Y.; Chen, R.; Chen, X.; Zeng, Z.; Ma, H.; Chen, S., Dipeptidyl Peptidase IVInhibitory Peptides Derived from Silver Carp (Hypophthalmichthys molitrix Val.) Proteins. J Agric Food Chem 2016, 64, 831-9.

24. Huang, S.-L.; Jao, C.-L.; Ho, K.-P.; Hsu, K.-C., Dipeptidyl-peptidase IV inhibitory activity of peptides derived from tuna cooking juice hydrolysates. Peptides 2012, 35, 114-121.

25. Li-Chan, E. C.; Hunag, S. L.; Jao, C. L.; Ho, K. P.; Hsu, K. C., Peptides derived from atlantic salmon skin gelatin as dipeptidyl-peptidase IV inhibitors. J Agric Food Chem 2012, 60, 973-8.

26. Hatanaka, T.; Inoue, Y.; Arima, J.; Kumagai, Y.; Usuki, H.; Kawakami, K.; Kimura, M.; Mukaihara, T., Production of dipeptidyl peptidase IV inhibitory peptides from defatted rice bran. Food Chem 2012, 134, 797-802.

27. Mojica, L.; de Mejía, E. G., Optimization of enzymatic production of anti-diabetic peptides from black bean (Phaseolus vulgaris L.) proteins, their characterization and biological potential. Food Funct 2016, 7, 713-27.

28. Velarde-Salcedo, A. J.; Barrera-Pacheco, A.; Lara-Gonzalez, S.; Montero-Moran, G. M.; Diaz-Gois, A.; Gonzalez de Mejia, E.; Barba de la Rosa, A. P., In vitro inhibition of dipeptidyl peptidase IV by peptides derived from the hydrolysis of amaranth (Amaranthus hypochondriacus L.) proteins. Food Chem 2013, 136, 758-764.

29. Lammi, C.; Zanoni, C.; Scigliuolo, G. M.; D'Amato, A.; Arnoldi, A., Lupin peptides lower low-density lipoprotein (LDL) cholesterol through an up-regulation of the LDL receptor/sterol 
312

313

314

315

316

317

318

319

320

321

322

323

regulatory element binding protein 2 (SREBP2) pathway at HepG2 cell line. J Agric Food Chem 2014, 62, 7151-9.

30. Lammi, C.; Zanoni, C.; Arnoldi, A., Molecular characterization of the hypocholesterolemic mechanism of action of IAVPGEVA, IAVPTGVA, and LPYP, three peptides from soy glycinin, in HepG2 cells. $J$ Funct Foods 2015, 14, 469-478.

31. Lammi, C.; Zanoni, C.; Arnoldi, A.; Vistoli, G., Two peptides from soy $\beta$-conglycinin induce a hypocholesterolemic effect at HepG2 cells by a statin-like mechanism: Comparative in vitro and in silico modeling studies. J Agric Food Chem 2015, 63, 7945-7951.

32. Amigo-Benavent, M.; Clemente, A.; Caira, S.; Stiuso, P.; Ferranti, P.; del Castillo, M. D., Use of phytochemomics to evaluate the bioavailability and bioactivity of antioxidant peptides of soybean $\beta$-conglycinin. Electrophoresis 2014, 35, 1582-9.

33. Lammi, C.; Aiello, G.; Vistoli, G.; Zanoni, C.; Arnoldi, A.; Sambuy, Y.; Ferruzza, S.; Ranaldi, G., A multidisciplinary investigation on the bioavailability and activity of peptides from lupin protein. J Funct Foods 2016, 10.1016/j.jff.2016.04.017.

34. Minkiewicz, P.; Dziuba, J.; Darewicz, M.; Bucholska, J.; Mogut, D., Evaluation of In Silico Prediction Possibility of Epitope Sequences Using Experimental Data Concerning Allergenic Food Proteins Summarised in BIOPEP Database. Pol J Food Nutr Sci 2012, 62, 151-157.

35. Lammi, C.; Zanoni, C.; Arnoldi, A.; Vistoli, G., Two peptides from soy beta-conglycinin induce a hypocholesterolemic effect in HepG2 cells by a statin-like mechanism: Comparative in vitro and in silico modeling studies. J Agric Food Chem 2015, 63, 7945-7951.

36. Pedretti, A.; Villa, L.; Vistoli, G., VEGA: A versatile program to convert, handle and visualize molecular structure on Windows-based PCs. J Mol Graph Model 2002, 21, 47-9. 
334 37. Biftu, T.; Sinha-Roy, R.; Chen, P.; Qian, X.; Feng, D.; Kuethe, J. T.; Scapin, G.; Gao, Y. D.; 335 Yan, Y.; Krueger, D.; Bak, A.; Eiermann, G.; He, J.; Cox, J.; Hicks, J.; Lyons, K.; He, H.; Salituro, 336 G.; Tong, S.; Patel, S.; Doss, G.; Petrov, A.; Wu, J.; Xu, S. S.; Sewall, C.; Zhang, X.; Zhang, B.; 337 Thornberry, N. A.; Weber, A. E., Omarigliptin (MK-3102): a novel long-acting DPP-4 inhibitor for 338 once-weekly treatment of type 2 diabetes. J Med Chem 2014, 57, 3205-12.

339 38. Korb, O.; Stützle, T.; Exner, T. E., Empirical scoring functions for advanced protein-ligand 340 docking with PLANTS. J Chem Inf Model 2009, 49, 84-96.

341 39. Pieter, B. J.-W. Protein hydrolysate enriched in peptides inhibiting DPP-IV and their use. WO $342 \quad 2006 / 068480200,2006$. 
344

345

346

347

348

349

350

351

352

353

354

355

\section{CAPTIONS OF FIGURES}

Figure 1. DPP IV inhibitory activities of Soy 1-3 and Lup 1-3. Each peptide was tested at a final concentration of $100 \mu \mathrm{M}$, in parallel with the positive control, sitagliptin, at a final concentration of $0.1 \mu \mathrm{M}$. Bars represent the averages \pm SEM of 3 independent experiments in triplicate. ns: not significant and $* * * P<0.0001$ versus the enzyme activity.

Figure 2. Dose-response curves of the inhibitory action of Soy 1 and Lup 1 peptides on DPP IV. The estimated $\mathrm{IC}_{50}$ values are equal to $106 \mu \mathrm{M}$ and $228 \mu \mathrm{M}$, respectively. The data points represent averages \pm SEM of three independent experiments in triplicate.

Figure 3. Key ionic interactions stabilizing the putative complex between Soy 1 (shown by a blue tube) and DPP IV. The displayed protein residues are also involved in key interactions with Omarigliptin as seen in the utilized resolved DPP IV structure. 
Table 1. Soy and lupin peptides.

\begin{tabular}{|c|c|c|c|c|}
\hline Plant & Parent protein & $\begin{array}{c}\text { Enzyme of } \\
\text { digestion }\end{array}$ & Sequence & Entry \\
\hline \multirow{2}{*}{ Soybean } & glycinin & pepsin & IAVPTGVA & Soy 1 \\
\cline { 2 - 5 } & $\beta$-conglycinin & pepsin/pancreatin & YVVNPDNDEN & Soy 2 \\
\cline { 2 - 6 } & $\beta$-conglycinin & pepsin/pancreatin & YVVNPDNNEN & Soy 3 \\
\hline \multirow{2}{*}{ Lupin seed } & $\beta$-Conglutin & pepsin & LTFPGSAED & Lup 1 \\
\cline { 2 - 6 } & $\beta$-Conglutin & pepsin & LILPKHSDAD & Lup 2 \\
\cline { 2 - 6 } & $\beta$-Conglutin & trypsin & GQEQSHQDEGVIVR & Lup 3 \\
\hline
\end{tabular}

357

358

359

360 


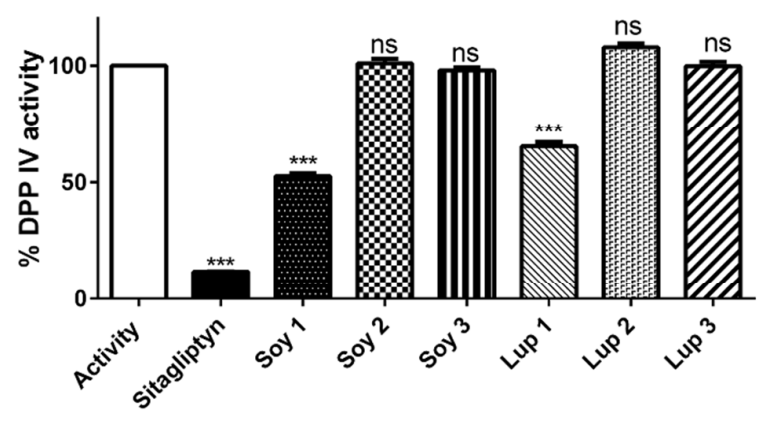

361

362 Figure 1

363

364

365

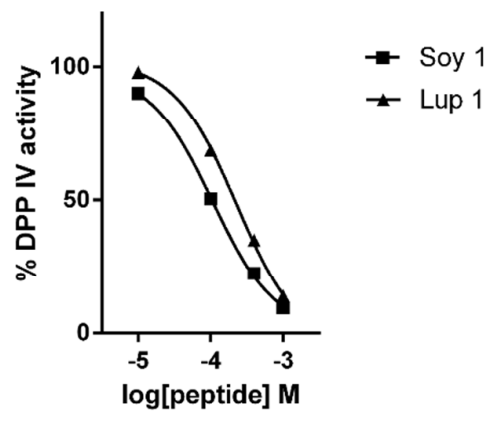

366 Figure 2

367

368

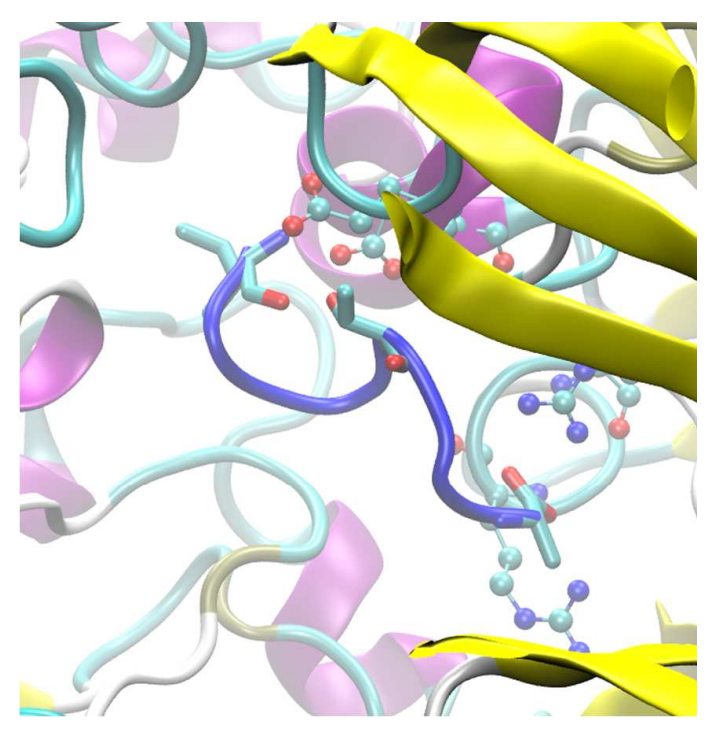

$371 \quad$ Figure 3 
20

372 TOC

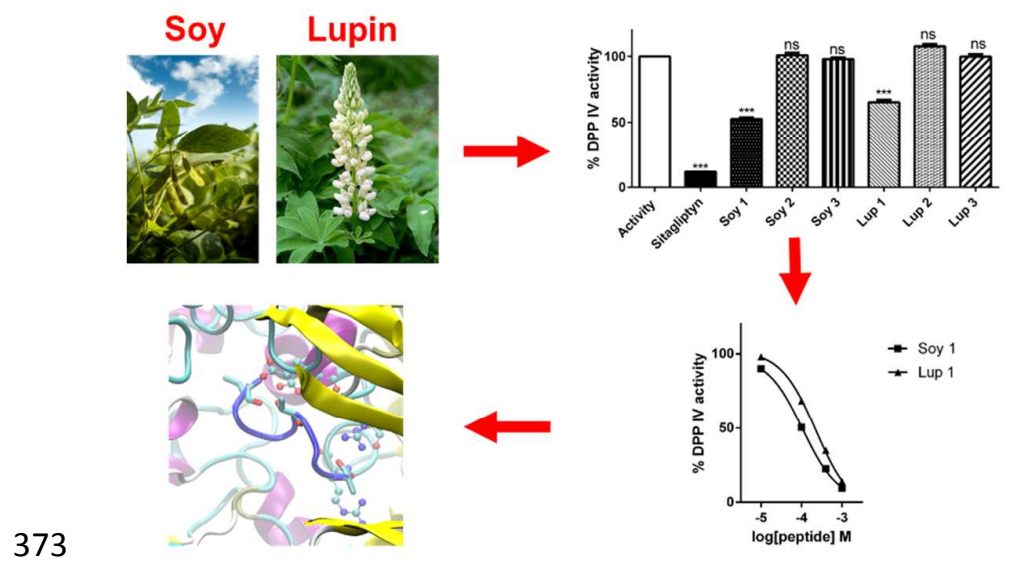

